\title{
28
}

\author{
DE \\ COLOPROCTOLOGIA
}

\author{
JULHO / SETEMBRO 2008
}

ARTIGOS ORIGINAIS

\section{Resultados do Tratamento do Câncer Colorretal (T4) Perfurado: Análise de 14 Pacientes Operados}

\author{
Treatment Outcomes from Perforated T4 Colorectal Cancer: \\ Analysis of 14 Resectable Cases
}

${ }^{1}$ SERGIO RENATO PAIS COSTA; ${ }^{2}$ RENATO ARIONI LUPINACCI
${ }^{1}$ Especialista em Cirurgia do Aparelho Digestivo e Cancerologia Cirúrgica - Médico Assistente do Serviço de Cirurgia Geral e Oncocirurgia do Hospital do Servidor Público Estadual de São Paulo - "Francisco Morato de Oliveira", Mestre em Cirurgia pela Universidade Federal de São Paulo; ${ }^{2}$ Especialista em Coloproctologia - Diretor do Serviço de Cirurgia Geral e Oncocirurgia do Hospital do Servidor Público Estadual de São Paulo - "Francisco Morato de Oliveira”, Doutor em Cirurgia pela Universidade Federal de São Paulo.

COSTA SRP; LUPINACCI RA. Resultados do Tratamento do Câncer Colorretal (T4) Perfurado: Análise de 14 Pacientes Operados. Rev bras Coloproct, 2008;28(3): 274-280.

RESUMO: Objetivo - Relatar série de 14 casos de câncer colorretal T4 complicado (perfuração) submetidos à cirurgia curativa, analisando a morbidade, mortalidade e sobrevida dos doentes. Métodos - Os dados completos de 14 pacientes submetidos a esse tipo de operação por adenocarcinoma colorretal T4 complicado entre 1999 e 2007 foram avaliados. Foram analisados os achados epidemiológicos e cirúrgicos. A sobrevida em longo prazo também foi avaliada. Resultados - A mortalidade pós-operatória foi de $14 \%(n=2)$. A morbidade global foi de $50 \%(n=7)$. Dez ressecções foram R0 (71 \%). Seis doentes apresentaram linfonodo comprometido $(43 \%)$ e todos faleceram de recurrência do tumor (4 - 19 meses). Seis doentes (n=6) estavam vivos sem recidiva (entre 18 e 70 meses/seguimento). A sobrevida global em cinco anos foi de 25\% (n=3). Conclusão -. A ressecção radical (R0) do câncer colorretal perfurado apresenta altos índices de morbidade e mortalidade. A despeito de uma elevada recurrência tumoral, esse procedimento pode oferecer controle da doença em longo prazo.

Descritores: Neoplasias Colorretais, Taxa de Sobrevida, Morbidade, Mortalidade, Resultados do Tratamento.

\section{INTRODUÇÃO}

A perfuração neoplásica no câncer colorretal é uma complicação incomum que envolve um quadro dramático que invariavelmente evolui para intervenção cirúrgica imediata. Nessa situação, tanto a morbidade quanto a mortalidade são extremamente elevadas, em virtude principalmente do quadro clínico de peritonite fecal que se instala (1-3). Índices de mor- talidade pós-operatória de 11 até $43 \%$ têm sido descritos, mesmo em casuísticas mais recentemente publicadas $(1,3,4-7)$. Não se trata de uma circunstância de fácil manejo terapêutico, haja vista que tanto a doença (tumor) quanto a complicação (perfuração) devem ser simultaneamente abordadas. Embora poucos estudos tenham sido publicados no que tange a essa singular ocorrência, geralmente seu prognóstico final tem sido considerado reservado devido a uma possível

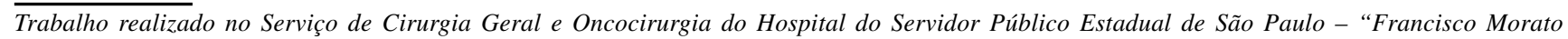
de Oliveira” - São Paulo - SP - Brasil. 
disseminação das células neoplásicas na cavidade abdominal $(4,5)$. Estatisticamente, trata-se de um evento relativamente raro, cuja incidência tem variado de 2,3 a 9\% dentre todos os casos de tumores colorretais operados em suas diferentes séries (1-4). Há controvérsia se uma operação radical deva ser realizada nessa condição, provavelmente pela chance de disseminação neoplásica. Em nosso meio carecem estudos em relação a esse grupo de doentes e baseado nesses achados realizamos um estudo retrospectivo cujo objetivo foi avaliar os resultados do tratamento de uma série de pacientes com câncer de colorretal perfurado submetidos à cirurgia de urgência no Hospital do Servidor Público Estadual de São Paulo, "Francisco Morato de Oliveira."

\section{MÉTODOS}

Foram analisados os prontuários de 17 doentes com tumor colorretal com perfuração neoplásica todos atendidos no Pronto-Socorro do Hospital do Servidor Público Estadual de São Paulo, no período de Setembro de 1999 a Setembro de 2007. Os pacientes foram operados sempre pela mesma equipe (Serviço de Cirurgia Geral e Oncocirurgia) desse hospital. Na presente casuística somente foram considerados doentes com perfuração no local da neoplasia primária. Dessa casuística inicial onde todos os doentes foram inicialmente submetidos à laparotomia exploradora, três foram excluídos da amostra, pois apresentavam doença à distância documentada ao inventário da cavidade abdominal. Desta maneira, 14 doentes foram selecionados para realização de uma cirurgia potencialmente curativa (R0) de urgência.

Oito eram do sexo masculino e seis do sexo feminino. A idade variou de 58 a 69 anos. Todos eram caucasianos. Em relação aos achados clínicos, todos apresentavam dor abdominal intensa, com defesa involuntária e sinais de irritação peritoneal. $\mathrm{O}$ tempo de instalação da dor e procura pelo pronto socorro variou de 5 a 48 horas, com mediana de 23 horas. Oito apresentavam sinais infecciosos como febre ou calafrios. A maioria desses doentes apresentava leucocitose $(n=10)$ e anemia $(n=8)$ sendo que três apresentavam ainda desnutrição (albumina sérica inferior a 3,5). Dois doentes apresentavam sinais de choque séptico à entrada no pronto-socorro.

Em relação à localização do tumor: nove apresentavam tumores no cólon enquanto cinco apresentavam tumores no reto. As características epidemiológicas e os achados laboratoriais estão representados respectivamente nas Tabelas 1 e 2.

Em todos os doentes foram realizados além do exame físico e dos exames laboratoriais, exames de imagem como do rx simples de abdome e tomografia computadorizada de abdome e pelve (Figura 1). Em onze doentes as cirurgias realizadas foram exclusivamente colorretais enquanto em três foram multiviscerais. Nas cirurgias exclusivamente colorretais a distribuição foi à seguinte: colectomia direita $(n=4)$, colectomia esquerda $(n=2)$, ressecção anterior alta $(n=5)$. Já em relação às cirurgias alargadas (todos com tumores de reto) houve a seguinte distribuição: em dois

Tabela 1 - Características epidemiológicas.

\begin{tabular}{ll}
$N=14$ & \\
\hline Mediana de Idade & 63 (58-69) \\
Sexo & \\
Masculino & 8 \\
Feminino & 6 \\
Doenças Associadas & \\
Hipertensão & 3 \\
Diabetes Mellitus & 1 \\
Obesidade & 1 \\
Sintomas & \\
Dor abdominal & 8 \\
Tenesmo & 4 \\
Fístula (reto-vaginal ou vesical) & 2 \\
Sangramento retal & 1 \\
ASA & \\
I & 8 \\
II-III & 6 \\
Localização das lesões & \\
Cólon Direito & 4 \\
Cólon Esquerdo-Sigmóide & 5 \\
Reto & 5 \\
\hline
\end{tabular}

Tabela 2 - Achados Laboratoriais.

$N=14$

\begin{tabular}{|c|c|}
\hline Hemoglobina & $(10,1-$ \\
\hline Leucócitos & $13560(9000-23450)$ \\
\hline Uréia & - 230) \\
\hline Creatinina & $(0,8-$ \\
\hline Albumina & $(2,1-$ \\
\hline
\end{tabular}




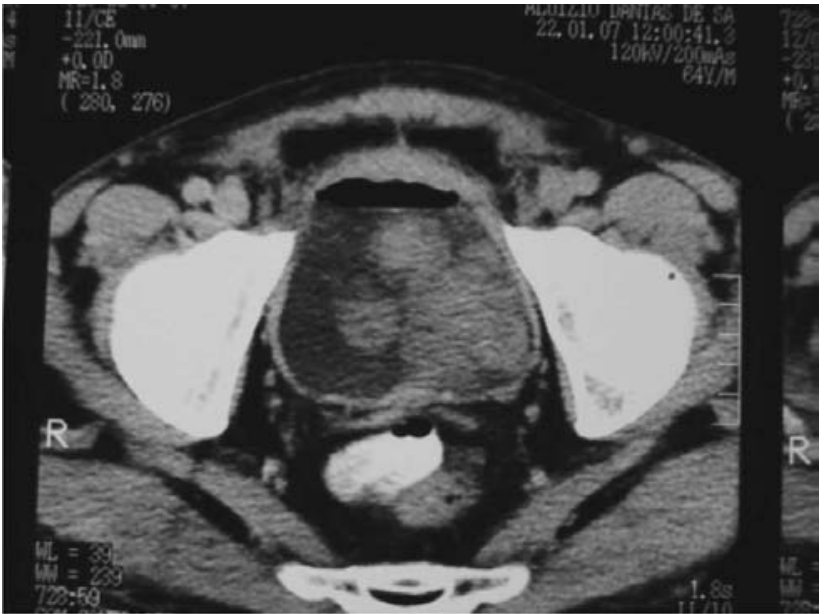

Figura 1 - TC de abdome mostrando grande lesão vegetante do reto infiltrando bexiga (nota-se ar no interior da bexiga).

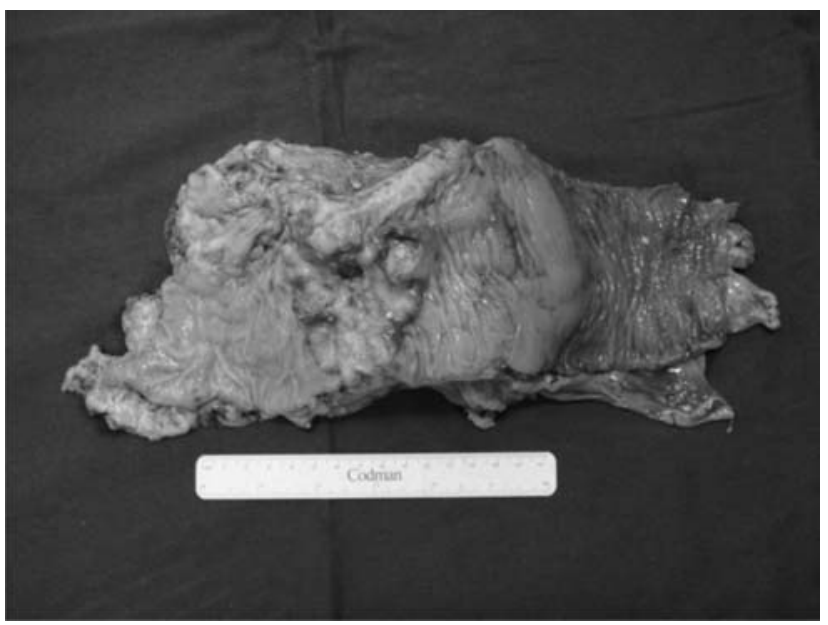

Figura 2 - Tumor perfurado no reto (visão anterior) - peça de ressecção alargada (exenteração total supra-elevadora).

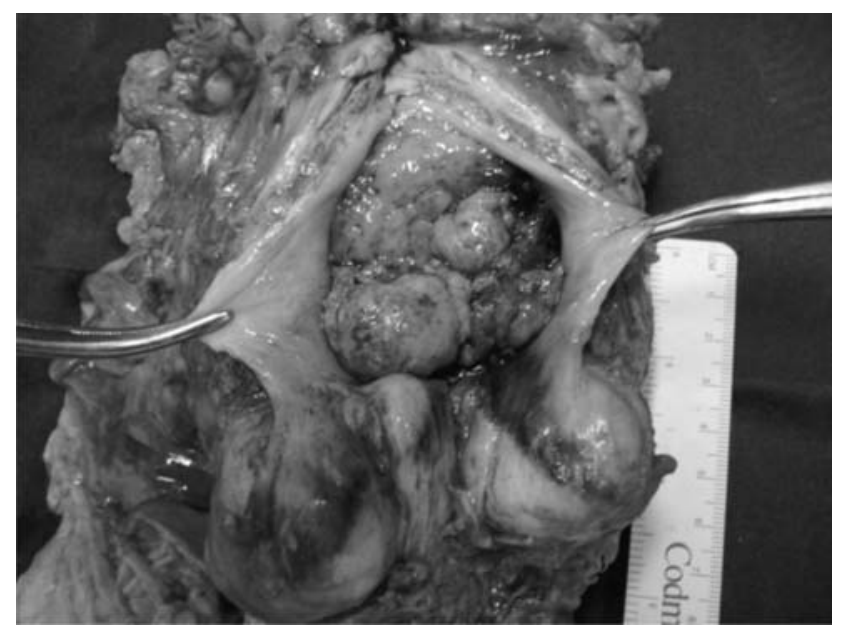

Figura 3 - Tumor de reto com Invasão do trígono vesical (visão posterior). doentes foi realizada exenteração pélvica supraelevadora (Figuras 2 e 3) enquanto em uma doente exenteração pélvica posterior. Apenas nos doentes submetidos à colectomia direita foi realizada anastomose primária, nos demais foi realizada uma reconstrução Tipo Hartmann. As reconstruções urinárias nas duas exenterações totais foram por meio de ureteroileostomia cutânea a Bricker. O tempo operatório variou de 180 a 400 minutos, com mediana de 200 minutos. Dez doentes foram transfundidos. O sangramento intra-operatório estimado variou de 200 a $3000 \mathrm{ml}$, com mediana de $800 \mathrm{ml}$. O tempo de internação variou de 13 a 75 dias, com mediana de 25 dias. No total dez doentes receberam tratamento adjuvante. Oito receberam tratamento quimioterápico exclusivo enquanto dois (ambos com tumores de reto) receberam tratamento combinado com quimioterapia e radioterapia adjuvantes.

Todos os pacientes foram acompanhados no pós-operatório conjuntamente pelo Serviço de Cirurgia Geral-Oncocirurgia e pelo Serviço de Oncologia Clínica do Hospital do Servidor Público Estadual de São Paulo. Exame físico, dosagem de antígeno carcinoembrionário (CEA), CA 19.9, RX de tórax, TC de abdome e pelve foram realizados a cada 3 meses até 2 anos após a cirurgia.

Após 2 anos, os pacientes foram avaliados a cada 6 meses. Uma colonoscopia anual foi realizada em cada paciente. O PET-SCAN foi realizado na dúvida quanto à recidiva tumoral nos últimos cinco doentes dessa série. Toda recidiva foi confirmada por exame de imagem (TC) e se possível confirmada por avaliação histológica.

\section{RESULTADOS}

A morbidade global da presente série foi de 50 $\%$. Sete doentes apresentaram pelo menos uma complicação pós-operatória. Quatro pacientes apresentaram complicações maiores e sofreram reintervenção cirúrgica, dois devido a múltiplos abscessos intra-abdominais, um por deiscência da anastomose urinária (ureteroileostomia cutânea) e um por evisceração (Tabela 3). A mortalidade global dessa série foi de $14 \%$ $(n=2)$, ambos os doentes que faleceram o fizeram por complicações infecciosas (ambos apresentavam choque séptico na entrada). Um foi submetido a uma ressecção multivisceral por câncer de reto perfurado e o outro uma hemicolectomia esquerda. Dentre todos os doentes operados, estes apresentavam os níveis mais 
baixos de albumina sérica e concomitantemente os mais altos de uréia, creatinina e de leucócitos de toda essa casuística. Contudo, ambos pertenciam à classificação I do escore ASA. Em dez doentes (71\%), as margens de ressecção foram livres de comprometimento neoplásico (Ressecção R0). Nos quatro restantes, as margens de secção apresentaram doença residual microscópica (Ressecção R1) sendo assim sua distribuição: reto $(n=2)$, sigmóide $(n=1)$ e cólon esquerdo $(n=1)$. Os órgãos invadidos pela neoplasia retal foram: bexiga $(\mathrm{n}=2)$, próstata $(\mathrm{n}=1)$, útero $(\mathrm{n}=1)$ e vagina $(\mathrm{n}=1) . \mathrm{O}$ grau histológico de diferenciação dos tumores ressecados também foi estudado e teve a seguinte distribuição: grau I $(n=4)$, grau II $(n=6)$ e grau III $(n=4)$. O número de linfonodos dissecados variou de 18 a 40 linfonodos, com mediana de 27 linfonodos. A avaliação histológica, todos os tumores eram T4 sendo que em seis doentes houve comprometimento linfonodal (43\%).

Durante o seguimento, seis doentes apresentaram recidivas $(50 \%)$ e faleceram da doença em um período que variou entre quatro e dezenove meses (cinco deles apresentavam comprometimento linfonodal enquanto um apresentava margem cirúrgica microscopicamente positiva, dois apresentavam tanto margem positiva quanto doença linfonodal). Todos os demais $(n=6)$ cujas margens cirúrgicas estavam livres e que coincidentemente não apresentavam comprometimento linfonodal estavam vivos e sem doença até o final do presente estudo em um período de seguimento que variou de 18 a 70 meses (mediana de seguimento de 60 meses). As características das recidivas e o tempo de sobrevida em longo prazo estão demonstrados na Tabela 4.

\section{DISCUSSÃO}

O índice de mortalidade pós-operatória no presente estudo em que pese elevado de $12 \%$ encontra-

Tabela 3 - Complicações e mortalidade.

Morbidade $50 \%(N=7)$

\begin{tabular}{lc}
\hline Abscesso intra-abdominal & 2 \\
Fístula Urinária & 1 \\
Evisceração & 1 \\
Pneumonia & 2 \\
Ìleo Prolongado & 1 \\
Reoperação & $28 \%(\mathrm{n}=4)$ \\
Mortalidade & $14 \%(\mathrm{n}=2)$ \\
\hline
\end{tabular}

se entre os valores previamente descritos na literatura que tem variado de 12 até $43 \%$ (1-6). A mortalidade pós-operatória nessa situação apresenta índices equivalentes às demais perfurações colorretais de etiologia benigna e tem sido muito mais associada à peritonite fecal e suas repercussões do que propriamente a neoplasia em si (7). Diversos fatores prognósticos têm sido associados à maior mortalidade na presença de peritonite entre eles os mais importantes tem sido: presença de hipotensão (choque) tanto pré quanto pósoperatória, nível elevado de creatinina sérica, ASA e escore APACHE II elevados $(5,7)$. Embora no presente estudo não tenha sido realizado uma avaliação dos critérios prognósticos pela pequena casuística apresentada, coincidentemente ambos doentes que faleceram apresentavam choque na entrada bem como altos índices de creatinina sérica em concordância aos estudos supracitados. Curiosamente, esses doentes também apresentavam os mais elevados índices de leucocitose dessa série, contudo não encontramos na literatura consultada leucocitose como fator isolado de mortalidade pós-operatória. Em que pese à classificação da ASA tenha sido descrita previamente como fator preditivo significante de mortalidade pós-operatória em uma análise multivariada (7), no presente estudo não foi observado esse achado (dois óbitos - ASA I). Embora um doente que tenha falecido nessa presente série tenha sido submetido a uma ressecção alargada e paralelamente saibamos que as ressecções multiviscerais apresentem alta mortalidade principalmente quando envolvem o reto (8-10), pelo nível de evidência atual não podemos inferir que uma ressecção multivisceral seja por si só fator prognóstico independente de mortalidade pós-operatória.

Historicamente, o câncer colorretal perfurado tem sido considerado de péssimo prognóstico devido à possibilidade de disseminação na cavidade peritoneal.

Tabela 4 - Características das recidivas e tempo de sobrevida.

\begin{tabular}{lc}
\hline Recidivas & $50 \%(\mathrm{n}=6)$ \\
Pelve & 1 \\
Peritônio & 2 \\
Fígado & 3 \\
Sobrevida Global & \\
Em 3 anos & $33 \%(\mathrm{n}=4)$ \\
Em 5 anos & $25 \%(\mathrm{n}=3)$ \\
\hline
\end{tabular}


Paralelamente, tem sido frequentemente observado nesse tipo de apresentação clínica, a presença de neoplasia localmente avançada ou mesmo com disseminação à distância piorando em muito seu prognóstico final $(1,11)$. Fato esse observado também no presente estudo onde três doentes já apresentavam doença à distância a laparotomia e ainda outros três apresentavam tumores que invadiam estruturas contíguas sendo assim submetidos à ressecção multivisceral (20\%). A perfuração neoplásica foi reportada como sendo o único indicador significante de sobrevida livre de doença devido ao fato de que alguns autores tenham considerado que a dispersão de células tumorais na cavidade peritoneal pelo sítio de perfuração leve a implantação do tumor e sua posterior disseminação (2, 12). Entretanto, no que tange ao efeito da dispersão das células tumorais no câncer colorretal perfurado no prognóstico, Lehnert et al (13) relataram que células tumorais livres e viáveis não foram demonstradas na cavidade peritonial de pacientes com tumores gastrointestinais perfurados e a eficiência metastática das células cancerígenas que poderiam se implantar durante a perfuração era incerta na presença de peritonite. Estudos recentes têm demonstrado que a sobrevida dos pacientes submetidos à ressecção completa de tumores perfurados tem sido similar a tumores não complicados e que a presença isolada de perfuração não seria indicador de prognóstico reservado $(2,4)$.

Lee et al (4) observaram em seu estudo que a disseminação de células tumorais na presença de peritonite não alterou o prognóstico final dos enfermos e encontraram índices de sobrevida global em cinco anos de 57,8\% e de sobrevida livre de doença de 52,8 $\%$. Resultados similares foram encontrados por outros autores na presença de uma ressecção R0 para tumores perfurados, onde a sobrevida tem variado de 40 a $51 \%$ em cinco anos $(2,13)$. A sobrevida encontrada no presente estudo de $25 \%$ em cinco anos foi menor do que a descrita, contudo vale a pena ressaltar que foi observado um número excessivamente alto de doentes com comprometimento linfonodal (43\%). Sabidamente, comprometimento linfonodal é um dos principais fatores prognósticos negativos em diversos estudos previamente publicados de tumores colorretais inclusive séries de tumores $\mathrm{T} 4(4,8,10,12)$. Talvez essa alta prevalência de doentes com linfonodos comprometidos possa ter diminuído a sobrevida global em longo prazo dessa série haja vista que cinco doentes com linfonodos positivos faleceram de recidiva da doença enquanto seis que não apresentavam comprometimento linfonodal não apresentaram recidiva inclusive três deles que sobreviveram mais que com cinco anos. Esse resultado vai de encontro ao estudo recentemente publicado por Lee et al. (4) onde esses autores relacionaram uma longa sobrevida em tumores perfurados T4 a um tratamento cirúrgico agressivo com alto índice de ressecção R0 (sem resíduo macroscópico). Esses autores relataram inclusive que se a peritonite fosse adequadamente manejada, uma ressecção R0 acarretaria em sobrevida similar em tumores perfurados e tumores não complicados, alegando que a presença de perfuração não tem um significado necessariamente de neoplasia incurável desde que adequadamente tratada.

Paralelamente, ressecções paliativas têm apresentado resultados em longo prazo desalentadores, fato este conhecido de longa data em diversos trabalhos previamente publicados $(4,8,10,12,13)$ sinalizando que mesmo com peritonite uma ressecção radical mesmo que multivisceral é a única chance de sobrevida em longo prazo (4) como foi possível também de se observar no presente estudo.

Assim como Lee et al (4), observou-se uma mediana de idade dos doentes elevada no presente estudo (63 anos) bem como uma maior tendência de perfuração neoplásica nos segmentos distais do cólon ou mesmo reto $(71 \%)$. No presente estudo $100 \%$ dos casos foi devido à perfuração no sítio primário do tumor similar ao descrito por outros autores o que leva a se considerar que a perfuração pode devido à necrose do tumor e que a perfuração pode ocorrer sem obstrução (4).

Os procedimentos operatórios mais comumente realizados para perfurações colorretais têm sido as ressecções tipo Hartmann ou ressecção e anastomose com colostomia proximal em alça (4). Mais recentemente, alguns estudos têm sugerido que a ressecção e anastomose primária podem ser tão satisfatórias para as perfurações do cólon (direito ou esquerdo) quanto às operações estagiadas (14-15). Embora exista certa tendência na literatura a adotar com mais liberdade esta conduta, na presente casuística devido as condições locais não satisfatórias na maioria dos doentes (peritonite, sepsis) associado ainda presença de doenças clínicas e de idade avançada levou a considerar mais frequentemente cirurgias estagiadas principalmente em dois estágios (Hartmann).Um tempo cirúrgico mais prolongado ou mesmo uma fístula em uma 
Rev bras Coloproct Julho/Setembro, 2008
Resultados do Tratamento do Câncer Colorretal (T4) Perfurado: Análise de 14 Pacientes Operados

Sérgio Renato Pais Costa e Cols.
Vol. 28 $\mathbf{N}^{\circ} 3$ anastomose poderiam ter elevado a morbi-mortalidade nesta população de risco.

Em que pese o maior risco de cirurgias estagiadas, a maioria dos doentes da presente amostra era de idosos que sabidamente apresentam uma maior adaptação frente a uma colostomia definitiva. Fato esse que foi possível de ser observado no presente estudo onde em oito doentes com colostomia, somente um foi submetido à reconstrução de trânsito (o indivíduo mais jovem da casuística), todos os demais se recusaram à realização de tal procedimento quando foram abordados os riscos de uma nova intervenção. Em contrapartida, assim como a maioria dos autores, nas ressecções a direita houve uma grande preferência à anastomose imediata apresentando inclusive morbidade nula desses procedimentos (16-20).

\section{CONCLUSÃO}

A perfuração neoplásica no tumor colorretal é uma circunstância rara, porém de alta mortalidade. A ressecção radical da neoplasia (R0) nessa situação, embora apresente elevados índices tanto de morbidade quanto de mortalidade e uma ampla tendência à recidiva pode conferir um controle da neoplasia em longo prazo.

\footnotetext{
ABSTRACT: Aim - To report on a series of 14 patients with a complicated T4 colorectal cancer (perforated) and describe the outcomes (morbidity, mortality and long-term survival) following curative surgery. Methods - Complete follow-up data were available on 14 patients who underwent curative surgery from perforated colorectal adenocarcinoma between 1999 and 2007. Both epidemiological and surgical findings were analyzed. Long-term survival was evaluated too. Results - The postoperative mortality was $14 \%(n=2)$. The overall morbidity was $50 \%(n=7)$. Ten resections were $R 0$ (71 \%).Lymph node involvement was present in six patients $(43 \%)$, and all of them died due to tumor recurrence (4-19 months). Six patients (50\%) were alive without tumor recurrence (between 18 and 70 months/follow-up). The overall 5-year survival rate was $25 \%$ ( $\mathrm{n}=3$ ). Conclusion -. Radical resection (R0) of pefurated colorectal cancer presents high rates of morbidity and mortality. In spite of its high rate of tumor recurrence, this procedure may offer long-term disease control.
}

Key words: Colorectal Neoplasms, Intestinal Perfuration, Survival rate, Morbidity, Mortality, Treatment outcomes.

\section{REFERÊNCIAS}

1. Mandava N, Kumar S, Pizza WF, Aprile IJ. Perforated colorectal carcinomas. Am J Surg 1996; 172: 236 -8.

2. Chen HS, Sheen-Chen SM. Obstruction and perforation in colorectal adenocarcinoma: an analysis of prognosis and current trends. Surgery 2000; 127: 370-6.

3. Tobaruela E, Camunas J, Enriquez-Navascues JM, Diez M, Martin A, Ratia T et al. Medical factors in the morbidity and mortality associated with emergency colorectal surgery. Rev Esp Enferm Dig 1997; 89: 13-22.

4. Lee IK, Sung NY, Lee YS, Lee SC, Kang WK, Cho HM et al. The survival rate and prognostic factors in 26 perforated colorectal cancer patients. Int J Colorectal Dis 2006; 22 (5): 467-73.

5. Anwar MA, D'Souza F, Coulter R, Memon B, Khan IM, Memon MA. Outcome of acutely perforated colorectal cancers: Experience of single district general hospital.Surg Oncol 2006; 15 (2): 91-6.

6. Kyllonen LE. Obstruction and perforation complicating colorectal carcinoma. Acta Chir Scand 1987; 153: 607-14.
7. Horiuchi A, Watanabe Y, Doi T, Sato K, Yukumi S, Yoshida M, et al. Evaluation of prognostic factors and scoring system in colonic perfuration. World J Gastroenterol 2007; 13 (23): 3228-31.

8. Ike H, Shimada H, Yamaguchi S, Ichikawa Y, Fujii S, Ohki S. Outcome of total pelvic exenteration for primary rectal cancer. Dis Colon Rectum 2001; 46: 474-80.

9. Costa SRP, Antunes RCP, Paula RP, Pedroso MA, Farah JFM, Lupinacci RA. A exenteração pélvica no tratamento do câncer de reto T4: experiência de 15 casos operados. Arq Gastroenterol 2007; 44 (4): 284-8.

10. Costa SRP, Teixeira ACP, Lupinacci RA. A Exenteração Pélvica para o Câncer de Reto: Avaliação dos Fatores Prognósticos de Sobrevida em 27 Pacientes Operados. Rev bras Coloproctol 2008; 28 (1): 7-18.

11. Willet C, Tepper JE, Cohen A, Orlow E, Welch C. Obstructive and perforative colonic carcinoma: patterns of failure. J Clin Oncol 1985; 3: 379-84.

12. Steinberg SM, Brakin JS, Kaplan RS, Stablein DM. Prognostic Factors of colon tumors. Cancer 1986; 57: 1866-70.

13. Kagda FHY, Nyam DCNK, Ho YH, Eu KW, Leong AFPK, Seow-Choen F. Surgery may be curative for patients with a 
localized perforation of rectal carcinoma. Br J Surg 1999; 86: 1448-50.

14. Sasaki LS, Allaben RD, Golwala R, Mittal VK. Primary repair of colon injuries: a prospective randomized study. J Trauma 1995; 39: 895-901.

15. Gonzales RP, Merlotti GJ, Holevar MR. Colostomy in penetrating colon injury: is it necessary? J Trauma 1996; 41: 271-5.

16. Biondo S, Perea MT, Rague JM, Jaurrieta E. One-stage procedure in non-elective surgery for diverticular disease complications. Colorectal Dis 2001; 3: 42-5.

17. Park UC, Chung SS, Kim KR, Seong MK, Yoon WH, Kim YJ et al. Single-stage procedure with intraoperative colonoscopy and colonic irrigation in patients with obstructing left-sided colonic cancer. Int J Colorectal Dis 2004; 19: 487-92.
18. Radcliffe AG, Dudley HA. Intraoperative antegrade irrigation of the large intestine. Surg Gynecol Obstet 1983; 156: 721-3.

19. Tan SG, Nambiar R, Rauff A, Ngoi SS, Goh HS. Primary resection and anastomosis in obstructed descending colon due to cancer. Arch Surg 1991; 126: 748-51.

20. Murray JJ. Non-elective colon resection: alternative to multistage resections. Surg Clin North Am 1991; 71: 1187-94.

\section{Endereço para correspondência:} SERGIO RENATO PAIS COSTA

Instituto de Oncologia São Paulo

Av. Pacaembu, 1400

CEP: 01234-000

Tel: 11 - 3666-2299

E-mail:sergiorenatopais@ig.com.br 